\title{
SoftWipe - a tool and benchmark to assess scientific software quality
}

\author{
Adrian Zapletal $^{*, 1,2}$, Dimitri Höhler ${ }^{*, 1,2}$, Carsten Sinz $^{2}$, and Alexandros Stamatakis ${ }^{1,2}$ \\ ${ }^{1}$ Computational Molecular Evolution group, Heidelberg Institute for Theoretical Studies, Heidelberg, Germany \\ ${ }^{2}$ Institute for Theoretical Informatics, Karlsruhe Institute of Technology, Karlsruhe, Germany
}

\begin{abstract}
Scientific software from all areas of scientific research is pivotal to obtaining novel insights. Yet the quality of scientific software is rarely assessed, even though it might lead to incorrect scientific results in the worst case. Therefore, we have developed an open source tool and benchmark called SoftWipe, that provides a relative software quality ranking of 51 computational tools from diverse research areas. SoftWipe can be used in the review process of software papers and to inform the scientific software selection process.
\end{abstract}

\section{Correspondence: alexandros.stamatakis@h-its.org}

Scientific software (henceforth called software) has become pivotal for discoveries in almost all research fields, including emerging areas such as the digital humanities. The importance of software has become evident to the broad public by the plethora of computational tools (henceforth called tools) used to analyze SARS-CoV-2 data, for instance. However, due to the conditions under which software is typically being developed, such as, lack of sustainable funding for maintaining widely used tools, lack of time, and a lack of formal training in computer programming and software engineering, software quality exhibits a large variance. More importantly, software quality is typically not taken into account in the software development, software paper review, or in the tool selection process, for instance, when two computational tools provide analogous functionality. This is particularly worrisome as political decisions are currently partially based on such tools for handling the SARS-CoV-2 pandemic.

The term 'software quality' is admittedly fuzzy, but offers a handle to perform largely automated analyses of scientific open source tools based on a set of objective criteria and measures. Such an automated analysis of numerous tools would be hard if one wanted to apply proper software verification techniques. When a tool exhibits 'bad' software quality, this does absolutely not imply that it is erroneous. However, it does imply that the probability for it to generate faults that might, in the worst case, lead to paper retractions or incorrect results obtained with the faulty tool is higher (based on results from the area of empirical software engineering $(1,2)$ ). See the introduction of (3) for a list of some high profile retractions and corrections because of software errors. For the above reasons, we strongly advocate that software quality should be assessed in the development, review, and tool selection process.

To this end, and based on our previous work (4) where we manually assessed the software quality of widely used tools

\footnotetext{
* equal contribution
}

for evolutionary biology, we introduce SoftWipe, a largely automated open source tool and benchmark for assessing the software quality of open source tools written in $\mathrm{C}$ or $\mathrm{C}++$. We envision that SoftWipe can be used by authors/developers (e.g., requiring them to provide their SoftWipe score with the submission), reviewers and researchers to integrate software quality into the review process and to raise awareness about software quality and engineering issues which are crucial for the success of computational science in general.

The SoftWipe score is a relative score over a number of software quality indicators (see further below and the supplement for a description of these indicators). The best-ranking tool per indicator receives a score of 10 while the worst ranking tool per indicator receives a score of 0 . Subsequently, we compute the average unweighted score per tool over all per indicator scores to obtain a global software quality ranking.

This global relative score might change over time as more tools are being added to the benchmark and will therefore be difficult to reference. We have therefore also devised an absolute fixed global score (see supplement for mathematical details) that does not change when further tools are added to the benchmark and that can therefore easily be referenced.

We use the following software quality indicators (normalized by average values per 1000 lines of code) to rate the tools: number of compiler, sanitizer, and static code analyzer warnings as generated by a variety of tools, number of assertions used, cyclomatic code complexity, indicators of bad programming style, and degree of code duplication. A detailed description of the quality indicators including a rationale for the inclusion of each one is provided in the supplement.

The current benchmark comprises 51 tools from a wide range of scientific fields such as, evolutionary biology, astrophysics, epidemiological simulation, hypergraph analysis, deep learning applied to DNA data, antigen typing, protein data structure mining, SAT (satisfiability) solvers, etc. The benchmark results are provided in Table 1 Note again that absolute fixed SoftWipe scores do not change when new tools are included in the benchmark, while relative scores do change (see supplement for details). A comprehensive table including all individual software quality indicators as well as descriptions of the respective tools is available online at https://github.com/adrianzap/softwipe/wi ki/Code-Quality-Benchmark.

One important observation is that the four top scoring tools have all been developed by researchers with formal training 


\begin{tabular}{|c|c|c|}
\hline program name & absolute score & relative score \\
\hline genesis & 8.6 & 8.8 \\
\hline hyperphylo & 8.6 & 8.6 \\
\hline kahypar & 8.4 & 8.5 \\
\hline candy-kingdom & 8.2 & 8.2 \\
\hline fastspar & 8.0 & 7.9 \\
\hline bindash-1.0 & 7.9 & 7.9 \\
\hline repeatscounter & 7.6 & 7.7 \\
\hline naf-1.1.0/unnaf & 7.6 & 7.5 \\
\hline naf-1.1.0/ennaf & 7.5 & 7.4 \\
\hline virulign-1.0.1 & 7.5 & 7.4 \\
\hline axe- 0.3 .3 & 7.5 & 7.5 \\
\hline ExpansionHunter & 7.4 & 7.5 \\
\hline glucose-3-drup & 7.1 & 7.0 \\
\hline raxml-ng & 7.0 & 7.0 \\
\hline dawg & 6.8 & 6.9 \\
\hline ntEdit-1.2.3 & 6.4 & 6.2 \\
\hline defor & 6.4 & 6.4 \\
\hline swarm & 6.2 & 6.2 \\
\hline lemon & 6.1 & 6.0 \\
\hline treerecs & 6.1 & 6.1 \\
\hline BGSA_CPU-1.0 & 6.0 & 5.4 \\
\hline IQ-TREE-2.0-rc1 & 6.0 & 5.7 \\
\hline dr_sasa_n & 5.9 & 6.0 \\
\hline emeraLD & 5.8 & 5.5 \\
\hline copmem- 0.2 & 5.6 & 5.7 \\
\hline samtools & 5.6 & 5.6 \\
\hline seq-gen & 5.5 & 5.6 \\
\hline dna-nn-0.1 & 5.3 & 5.2 \\
\hline $\mathrm{sf}$ & 5.2 & 5.2 \\
\hline cryfa-18.06 & 5.1 & 5.1 \\
\hline ngsLD & 5.1 & 5.0 \\
\hline HLA-LA & 4.9 & 4.5 \\
\hline iqtree1.6.10 & 4.9 & 4.9 \\
\hline prequal & 4.6 & 4.4 \\
\hline vsearch & 4.6 & 4.6 \\
\hline prank & 4.6 & 4.5 \\
\hline minimap & 4.5 & 4.4 \\
\hline phyml & 4.4 & 4.4 \\
\hline clustal & 4.2 & 4.3 \\
\hline mrbayes & 4.1 & 4.1 \\
\hline tcoffee & 4.1 & 4.2 \\
\hline crisflash & 4.1 & 4.0 \\
\hline gadget & 4.0 & 4.0 \\
\hline PopLDdecay & 3.9 & 3.8 \\
\hline cellcoal & 3.8 & 3.6 \\
\hline bpp & 3.8 & 3.6 \\
\hline $\mathrm{ms}$ & 3.7 & 3.7 \\
\hline mafft & 3.2 & 3.1 \\
\hline athena & 2.9 & 2.8 \\
\hline covid-sim-0.13.0 & 2.5 & 2.4 \\
\hline indelible & 1.4 & 1.0 \\
\hline
\end{tabular}

Table 1. Absolute fixed and relative SoftWipe scores of 51 computational tools from a wide range of scientific fields such as computer science, evolutionary biology, astrophysics, and bioinformatics. The three DNA sequence simulation tools dawg, seq-gen, and indelible that have similar functionality are highlighted in bold font. in pure computer science whereas the 10 worst scoring tools have all been developed by self-taught programmers without formal training in computer science.

For an example of how the benchmark can be used, let us consider the three evolutionary molecular sequence data simulators dawg, seq-gen, and indelible (highlighted in bold font in Table 1). These tools implement highly similar functionalities and models. Our benchmark clearly suggests the use of dawg, as it obtains a higher score than the alternative sequence simulators.

It is also noteworthy that the developers of the top three tools had access to a pre-release version of SoftWipe as they are members or collaborators of our lab and improved their tools to obtain higher SoftWipe scores. This highlights the utility of SoftWipe within a lab such as ours that predominantly develops computational tools, as it fosters a healthy competition among lab members to develop the bestscoring tool. In fact, the regular deployment and integration of SoftWipe in the development process has also helped to avoid a bug in at least one occasion.

Given the current SARS-CoV-2 pandemic it is also worth noting that the CovidSim tool obtains the second worst score among all 51 tools tested.

We are well aware of the fact that SoftWipe is likely to generate controversial debates, as software quality does neither induce lack of code correctness, nor an erroneous underlying scientific model. Nonetheless, in the current absence of any standard routines for assessing scientific software quality and based upon the results of empirical software engineering research that establishes a correlation between software quality and failure, SoftWipe represents a step forward, as it is easy to use and does not require a substantial investment of time. This does not only apply to the software submission and review process, but also to software development. SoftWipe can also help to objectify controversial discussions about software quality, as was the case with the CovidSim tool (see e.g., https://www. imperial.a c.uk/news/197875/codecheck-confirms-repr oducibility-covid-19-model-results). We are therefore convinced that a routine deployment of SoftWipe or analogous tools at various stages of the software development and publication process can substantially contribute to improving software quality across all scientific fields, raise general awareness about the importance of software quality in the respective research communities, and reduce the number of program faults.

\section{Methods}

The open source code of SoftWipe including the up-to-date benchmark and a user manual is available at: https://gi thub.com/adrianzap/softwipe/wiki.

\section{Acknowledgements}

Part of this work was funded by the Klaus Tschira foundation. We wish to thank Bernd Doser for initial discussions on 
bioRxiv preprint doi: https://doi.org/10.1101/2020.10.07.330621; this version posted October 15, 2020. The copyright holder for this preprint

(which was not certified by peer review) is the author/funder, who has granted bioRxiv a license to display the preprint in perpetuity. It is made available under aCC-BY-NC-ND 4.0 International license.

SoftWipe and Frédéric Mahé for useful comments on this manuscript.

\section{Bibliography}

1. L. C. Briand, J. Wust, S. V. Ikonomovski, and H. Lounis. Investigating quality factors in object-oriented designs: an industrial case study. In Proceedings of the 1999 International Conference on Software Engineering (IEEE Cat. No.99CB37002), pages 345-354, 1999.

2. C. Casalnuovo, P. Devanbu, A. Oliveira, V. Filkov, and B. Ray. Assert use in github projects. In 2015 IEEE/ACM 37th IEEE International Conference on Software Engineering, volume 1 , pages 755-766, 2015.

3. Greg Wilson, Dhavide A Aruliah, C Titus Brown, Neil P Chue Hong, Matt Davis, Richard T Guy, Steven HD Haddock, Kathryn D Huff, Ian M Mitchell, Mark D Plumbley, et al. Best practices for scientific computing. PLOS Biol, 12(1):e1001745, 2014.

4. Diego Darriba, Tomáš Flouri, and Alexandros Stamatakis. The State of Software for Evolutionary Biology. Molecular Biology and Evolution, 35(5):1037-1046, 01 2018. ISSN 07374038. doi: $10.1093 / \mathrm{molbev} / \mathrm{msy} 014$ 